\title{
TMPRSS2-ERG gene fusions are infrequent in prostatic ductal adenocarcinomas
}

\author{
Tamara L Lotan ${ }^{1}$, Antoun Toubaji ${ }^{1}$, Roula Albadine ${ }^{1}$, Mathieu Latour ${ }^{1}$, Mehsati Herawi ${ }^{1}$, \\ Alan K Meeker ${ }^{1,2}$, Angelo M DeMarzo ${ }^{1,2,3}$, Elizabeth A Platz ${ }^{2,3,4}$, Jonathan I Epstein ${ }^{1,2,3}$ and \\ George J Netto ${ }^{1,2,3, *}$ \\ ${ }^{1}$ Department of Pathology, Johns Hopkins Medical Institutions, Baltimore, MD, USA; ${ }^{2}$ Department of Urology, \\ Johns Hopkins Medical Institutions, Baltimore, MD, USA; ${ }^{3}$ Department of Oncology, Johns Hopkins Medical \\ Institutions, Baltimore, MD, USA and ${ }^{4}$ Department of Epidemiology, Johns Hopkins Bloomberg School of \\ Public Health, Baltimore, MD, USA
}

\begin{abstract}
Ductal adenocarcinoma of the prostate is an unusual subtype that may be associated with a more aggressive clinical course, and is less responsive to conventional therapies than the more common prostatic acinar adenocarcinoma. However, given its frequent association with an acinar component at prostatectomy, some have challenged the concept of prostatic ductal adenocarcinoma as a distinct clinicopathologic entity. We studied the occurrence of the TMPRSS2-ERG gene fusion, in $\mathbf{4 0}$ surgically resected ductal adenocarcinoma cases, and in their associated acinar component using fluorescence in situ hybridization. A group of 38 'pure' acinar adenocarcinoma cases matched with the ductal adenocarcinoma group for pathological grade and stage was studied as a control. Compared with the matched acinar adenocarcinoma cases, the TMPRSS2-ERG gene fusion was significantly less frequently observed in ductal adenocarcinoma (45 vs $11 \%$ of cases, $P=0.002$, Fisher's exact test). Here, of the ductal adenocarcinoma cases with the gene fusion, $75 \%$ were fused through deletion, and the remaining case was fused through translocation. The TMPRSS2-ERG gene fusion was also rare in the acinar component of mixed ductal-acinar tumors when compared with the pure acinar adenocarcinoma controls (5 vs $45 \%, P=0.001$, Fisher's exact test). In $95 \%$ of the ductal adenocarcinoma cases in which a concurrent acinar component was analyzed, there was concordance for presence/absence of the TMPRSS2-ERG gene fusion between the different histologic subtypes. In the control group of pure acinar adenocarcinoma cases, $59 \%$ were fused through deletion and $41 \%$ were fused through translocation. The presence of the TMPRSS2-ERG gene fusion in some cases of prostatic ductal adenocarcinoma supports the concept that ductal adenocarcinoma and acinar adenocarcinoma may be related genetically. However, the significantly lower rate of the gene fusion in pure ductal adenocarcinoma cases underscores the fact that genetic and biologic differences exist between these two tumors that may be important for future therapeutic strategies.
\end{abstract}

Modern Pathology (2009) 22, 359-365; doi:10.1038/modpathol.2008.236; published online 16 January 2009

Keywords: Prostatic adenocarcinoma; ductal adenocarcinoma; endometrioid; TMPRSS2-ERG; gene fusion

Ductal adenocarcinoma of the prostate is an unusual subtype, accounting in its pure form for $0.4 \%$ of all prostate cancer, and occurring as a mixed tumor with the more common prostatic acinar adenocarcinoma in up to $5 \%$ of all radical prostatectomy cases. $^{1,2}$ First described nearly 40 years ago, these tumors are characterized classically by large periurethral ductal structures filled with papillary fronds or cribriform proliferations lined by columnar

${ }^{*}$ Correspondence: Dr GJ Netto, MD, Johns Hopkins Medical Institutions, $401 \mathrm{~N}$ Broadway, Weinberg Building, Suite 2242, Baltimore, MD 21231, USA.

E-mail: gnetto1@jhmi.edu

Received 4 December 2008; revised and accepted 17 December 2008; published online 16 January 2009 epithelium. ${ }^{3}$ In contrast to the more common acinar adenocarcinoma, prostatic ductal adenocarcinomas are often grossly visible to urologists on cystoscopy because of their central location within the gland and their occasional protrusion from the prostatic verumontanum. ${ }^{1}$ Historically, clinical evidence supporting the distinction of ductal adenocarcinoma from acinar adenocarcinoma, has been the fact that the ductal adenocarcinoma cases are typically more aggressive tumors than their acinar counterparts, presenting at higher clinical stage and less responsive to traditional hormonal, radiation and radical surgical therapies in a handful of prior studies. ${ }^{4-7}$

However, since the early description of ductal adenocarcinoma, it has become clear that there is a more clinical overlap between ductal adenocarcinoma 
and acinar adenocarcinoma than was originally thought. In radical prostatectomy specimens, ductal adenocarcinoma occurs with a concurrent conventional acinar component in the majority of cases, making these mixed tumors much more common than pure ductal adenocarcinoma. ${ }^{5}$ Much like acinar adenocarcinoma, tumors with the morphologic features of ductal adenocarcinoma may occur in peripheral locations. Additionally, a number of recent studies have challenged early reports of the ductal adenocarcinoma's resistance to traditional therapeutic strategies. ${ }^{2,8}$ Given the temporal and clinical overlap between these two variants of prostate cancer, some investigators have questioned whether ductal adenocarcinoma represents a truly distinct clinicopathologic entity, or whether these tumors are simply a morphologic variant in the spectrum of conventional acinar adenocarcinoma. ${ }^{2}$

Recently, a bioinformatics approach uncovered a gene rearrangement present in $40-60 \%$ of conventional prostatic acinar adenocarcinoma cases, making it the most common rearrangement identified in human cancer to date. ${ }^{9}$ This rearrangement occurs between an androgen-regulated gene, TMPRSS2 (transmembrane protease serine 2, 21q22.3) and an ETS transcription factor family member, most commonly ERG (v-ets erythroblastosis virus E26 oncogene homolog, 21q22.2), resulting in a gene fusion product. $^{10}$ This gene fusion can occur through a small deletion on chromosome 21 (seen approximately in two thirds of the acinar cases) or through a translocation. ${ }^{11}$ In either type of the rearrangement, ERG is brought under the control of an androgen-regulated promoter and over expression of the protein ensues. Although the clinicopathologic significance of this genetic rearrangement has remained elusive, it is clear that TMPRSS2-ERG rearrangements are specific and sensitive for prostatic acinar adenocarcinoma. ${ }^{12}$ The rearrangement may also be seen in concurrent highgrade prostatic intraepithelial neoplasia lesions, suggesting that it is a clonal and early pathogenic event in prostatic acinar adenocarcinoma. ${ }^{13}$

Despite the numerous studies of TMPRSS2-ERG gene fusions in prostatic acinar adenocarcinoma, no studies to date have examined the occurrence of this rearrangement in prostatic ductal adenocarcinoma. Indeed, documentation of the TMPRSS2-ERG gene fusion in ductal adenocarcinoma might help resolve the continuing debate over whether prostatic ductal adenocarcinoma represents a truly distinct clinicopathologic entity. Here, we studied the rate of TMPRSS2-ERG gene fusions in 40 cases of ductal adenocarcinoma, and in a control group of pathologic grade- and stage-matched acinar adenocarcinoma cases by fluorescence in situ hybridization (FISH). We report that although the TMPRSS2-ERG fusions do occur in ductal adenocarcinoma, they are considerably less frequent in these tumors than in their acinar counterparts, suggesting that significant genetic and potential biologic differences may exist between these two prostate tumor types. Such differences may be important for guiding future treatments of prostatic ductal adenocarcinoma.

\section{Materials and methods}

\section{Tissue Selection}

A tissue microarray was constructed manually from 40 radical prostatectomy specimens with ductal adenocarcinoma, retrieved from the surgical pathology and consultation files of the Johns Hopkins Hospitals from 1984-2005. In 52\% (21/40) of the ductal adenocarcinoma cases, a concurrent acinar component was present and included in the tissue microarray. In each case, quadruplicate $0.6 \mathrm{~mm}$ cores were punched from the ductal adenocarcinoma component, the acinar component (when present) and the surrounding benign prostatic tissue, with up to 16 total cores from each patient represented on the array. The ductal and acinar adenocarcinoma components were considered geographically separate primaries when the ductal adenocarcinoma and acinar adenocarcinoma tumor foci were separated by at least $5 \mathrm{~mm}$ in all dimensions.

Fifteen 'pure' acinar adenocarcinoma cases (Gleason pattern 4) were also included on the ductal adenocarcinoma tissue microarray as an internal control group. To provide additional pure acinar adenocarcinoma controls, an additional 23 acinar adenocarcinoma cases were selected for matched pathologic stage from a second tissue microarray, constructed from a PSA-era prostatectomy cohort (cases dating from 1993 to 2000). Overall, the 38 pure acinar adenocarcinoma controls were matched with the ductal adenocarcinoma group for pathological stage $(39 \%$ pT2, $58 \%$ pT3) and grade $(68 \%$ Gleason 7, 31\% Gleason 8-9) (Table 1).

\section{Fluorescence In Situ Hybridization}

FISH, using a break-apart probe for $5^{\prime}$ and $3^{\prime} E R G$, was performed on the two tissue microarrays. Briefly, sections of $4 \mu \mathrm{m}$-thick, paraffin-embedded

Table 1 Clinicopathologic characteristics of ductal adenocarcinoma cases and acinar adenocarcinoma controls. $\mathrm{U}=$ Unknown

\begin{tabular}{lccc}
\hline $\begin{array}{l}\text { Total number of } \\
\text { evaluable cases }\end{array}$ & $\begin{array}{c}\text { Ductal cases } \\
38\end{array}$ & $\begin{array}{c}\text { Acinar controls } \\
38\end{array}$ & P-value \\
\hline $\begin{array}{l}\text { Overall Gleason score, } n(\%): \\
\text { U }\end{array}$ & $4(10 \%)$ & $0(0 \%)$ & \\
7 & $21(55 \%)$ & $26(68 \%)$ & 0.570 \\
8 & $11(29 \%)$ & $10(26 \%)$ & \\
9 & $2(5 \%)$ & $2(5 \%)$ & \\
& & & \\
Pathologic stage: & $6(16 \%)$ & $1(3 \%)$ & \\
TX & $13(34 \%)$ & $15(39 \%)$ & 0.912 \\
T2 & $13(34 \%)$ & $16(42 \%)$ & \\
T3A & $6(16 \%)$ & $6(16 \%)$ & \\
T3B & & & \\
\hline
\end{tabular}


tissue microarrays were baked at $56^{\circ} \mathrm{C}$ for $2 \mathrm{~h}$, then dewaxed and rehydrated using xylene and graded ethanol, respectively. Tissue microarrays were pretreated using Paraffin Pretreatment Reagent Kit III (Abbott Molecular Inc., Abbott Park, IL). Bacterial artificial chromosome (BAC) FISH probes were SpectrumGreen d-UTP direct-labeled BAC RP1195I21 for $5^{\prime} E R G$ and SpectrumOrange d-UTP directlabeled BAC RP11-476D17 for $3^{\prime} E R G$ (Nick transKit, Vysis, Abbott Park, IL). Tissue microarrays and BAC FISH probes were co-denatured at $94^{\circ} \mathrm{C}$ for $5 \mathrm{~min}$ and hybridized over night at $37^{\circ} \mathrm{C}$ in a humid chamber (StatSpin ThermoBrite, IRIS Inc, MA).

\section{Fish Interpretation}

FISH interpretation was performed by one of the two urologic pathologists (TLL and ML) with confirmation of positive cases by a third urologic pathologist (RA). For each case, a minimum of 50 cells was scored for the presence/absence of the TMPRSS2$E R G$ gene fusion through deletion or translocation as follows: a nucleus without ERG rearrangement shows two pairs of juxtaposed red and green signals (Figure 1, bottom row, right panel). A nucleus with ERG rearrangement through deletion shows absence of one or more green signals (Figure 1, bottom row, left panel), and a nucleus with rearrangement through translocation shows one or more pairs of the red and green signals split apart and separated spatially in different regions of the nucleus (Figure 2, bottom row, both panels). Any case with one of the above ERG signal abnormalities in $\geq 10 \%$ of the nuclei was scored as a fusion case and classified accordingly. Digitally scanned adjacent hematoxylin and eosin serial sections were available for side-by-side comparison with the FISH image to localize tumor cells, and a Gleason grade was assigned to each sampled core. Five cases of benign prostatic epithelium were scored on each tissue microarray as a negative control.

FISH scoring was conducted using a 100x oil immersion lens on an Olympus AX-70 fluorescence microscope (Olympus, Center Valley, PA) equipped with appropriate filters. For photomicrographs, images were captured using a Nikon E400 fluorescence microscope equipped with a Nikon DXM1200 camera (Nikon Instruments, Melville, NY) and the SPOT Advanced digital imaging software (Diagnostic Instruments, Inc., Sterling Heights, MI).

\section{Statistical Analysis}

The findings were analyzed using the Stata 9.2. (StataCorp, College Station, TX) software package. An appropriate pathologic grade and stage matching of the ductal adenocarcinoma cases and the acinar adenocarcinoma controls was tested using the Kruskal-Wallis test for non-parametric one-way analysis of variance by ranks. Fisher's exact test

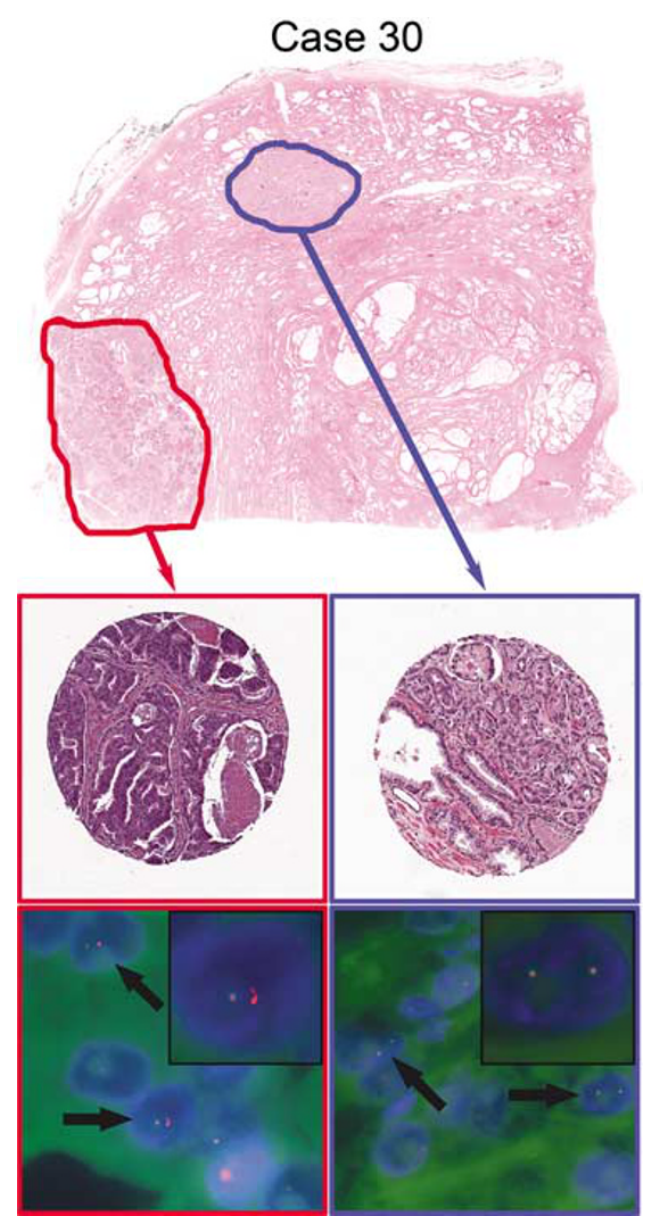

Figure 1 Top panel: low-power photomicrograph of the H\&E stained radical prostatectomy slide with ductal adenocarcinoma focus circled in red and acinar adenocarcinoma focus circled in blue (Case 30). The ductal and acinar tumors are separated spatially and appear to be discrete tumor foci. Middle panels: digitally scanned images of an H\&E stained section of the $0.6 \mathrm{~mm}$ cores punched for the tissue microarray from each tumor area $(40 \times$ magnification). Bottom panels: ERG break-apart FISH images from ductal and acinar tumor foci (all $1000 \times$ magnification). Case 30 shows the TMPRSS2-ERG fusion through deletion in the ductal adenocarcinoma (left panel), with one juxtaposed red-green (yellow) signal in each nucleus and absence of the second green signal (arrows, inset). The acinar component of Case 30 (right panel) shows no evidence of TMPRSS2-ERG fusion with two pairs of juxtaposed red-green signals in each nucleus (arrows, inset).

was used to compare frequency of the TMPRSS2$E R G$ fusion in ductal adenocarcinoma cases $v s$ acinar adenocarcinoma controls.

\section{Results}

Here, of the 40 ductal adenocarcinoma cases, 38 were evaluable by FISH for $E R G$ gene rearrangements (95\%). Overall, 11\% (4/38) of the ductal adenocarcinomas showed the TMPRSS2-ERG gene fusion, with $75 \%$ (3/4) showing the deletion and $25 \%$ (1/4) showing the translocation (Table 2, 


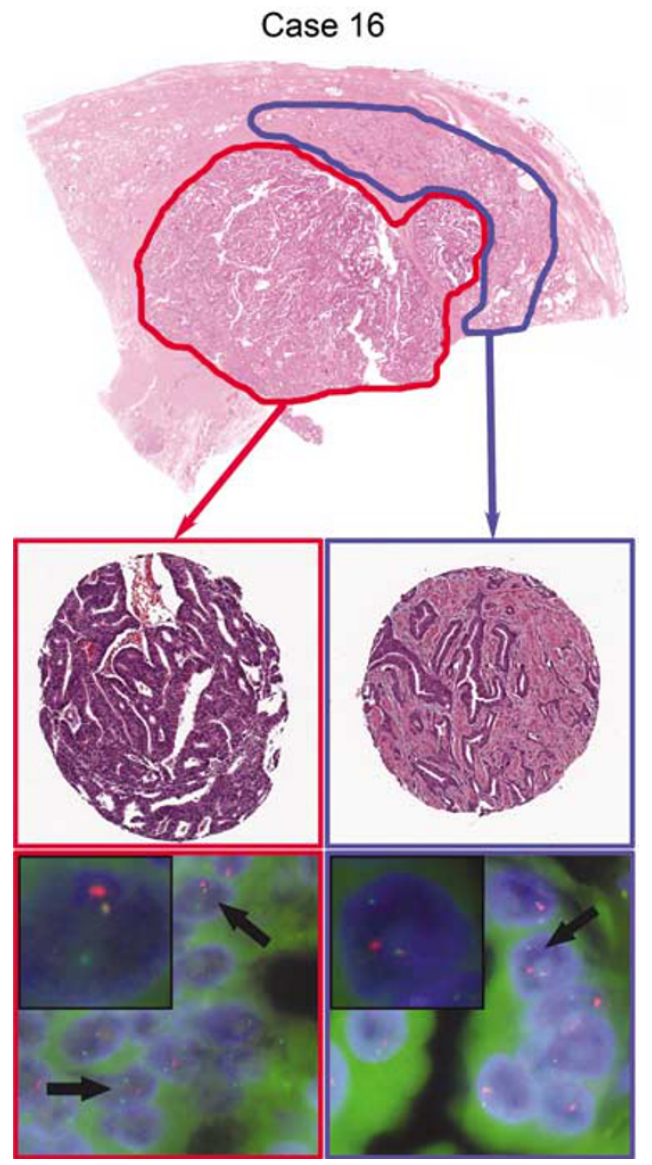

Figure 2 Top panel: low-power photomicrograph of the H\&E stained radical prostatectomy slide with ductal adenocarcinoma focus circled in red and acinar adenocarcinoma focus circled in blue (Case 16). The ductal and acinar adenocarcinoma foci are commingled spatially and likely represent a single primary tumor. Middle panels: digitally scanned images of an H\&E stained section of the $0.6 \mathrm{~mm}$ cores punched for the tissue microarray from each tumor area $(40 \times$ magnification). Bottom panels: ERG break-apart FISH images from ductal and acinar tumor foci (all $1000 \times$ magnification). Case 16 shows the TMPRSS2-ERG fusion through translocation in both the ductal and acinar tumor foci, with one juxtaposed red-green (yellow) signal in each nucleus and the second pair of red and green signals split apart and separated spatially in different regions of the nucleus (arrows, inset).

Figures 1 and 2). No cases of duplication/polyploidy of the fusion were observed in the ductal adenocarcinoma group. In $55 \%(21 / 38)$ of the evaluable ductal adenocarcinoma cases, a concurrent acinar component was present for evaluation as well. Although represented on separate cores in the tissue microarray, the ductal adenocarcinoma and acinar adenocarcinoma components were geographically separate primaries (separated by at least $5 \mathrm{~mm}$ in all dimensions), in the radical prostatectomy specimen in only $19 \%(4 / 21)$ of the cases (Figure 1). In $67 \%$ $(14 / 21)$ of the cases, the two morphologies commingled and appeared to represent a single primary tumor (Figure 2), and in $14 \%$ of the cases $(3 / 21)$, it was not possible to tell whether the components were separated spatially. In all the 21 cases, the sampled acinar component consisted primarily of
Table 2 Frequency of TMPRSS2-ERG rearrangement in ductal adenocarcinoma cases compared to pure grade- and stagematched acinar adenocarcinoma controls

\begin{tabular}{lccc}
\hline & Ductal cases & Acinar controls & P-value \\
\hline Total cases & 38 & 38 & \\
Rearrangement & $4(11 \%)$ & $17(45 \%)$ & 0.002 \\
Deletions & $3(75 \%)$ & $9(53 \%)$ & NA \\
Double deletion & $0(0 \%)$ & $1(6 \%)$ & NA \\
Translocation & $1(25 \%)$ & $7(41 \%)$ & NA \\
\hline
\end{tabular}

poorly formed or cribriform glands diagnostic of Gleason pattern 4 carcinoma (Gleason score 7-8). As seen in the ductal adenocarcinoma component, TMPRSS2-ERG gene fusion was also rare in the acinar component of mixed ductal-acinar adenocarcinomas $(1 / 21$ cases, $5 \%)$. In $95 \%$ of the ductal adenocarcinoma cases in which a concurrent acinar component was available for analysis (20/21), there was concordance for presence/absence of the TMPRSS2-ERG gene fusion between the different histologic subtypes (Table 3). The only discordant case (Case 30, Table 3, Figure 1) showed gene fusion through deletion in the ductal adenocarcinoma component and had no evidence of gene rearrangement in the cribriform acinar adenocarcinoma component. The ductal adenocarcinoma and acinar adenocarcinoma components appeared to be spatially separate primaries in this case (Table 3, Figure 1). In $95 \%(19 / 20)$ of the concordant cases, no gene rearrangement was detected, whereas in the remaining concordant case, a gene fusion through translocation was detected in the ductal adenocarcinoma as well as in the poorly formed glands of the acinar adenocarcinoma (Case 16, Table 3, Figure 2). In this case, the ductal adenocarcinoma and acinar adenocarcinoma components were commingled spatially.

Here, in the control group of 38 grade- and stagematched pure acinar adenocarcinoma cases, $45 \%$ (17/38) had the gene fusion with 53\% (9/17) showing the deletion, $6 \%(1 / 17)$ showing a duplicated deletion (deletion with polyploidy) and 41\% (7/17) showing the translocation (Table 2). All sampled tumor cores from the acinar adenocarcinoma control cases showed predominantly poorly formed or cribriform carcinoma glands and all were graded as primary Gleason pattern 4 carcinoma (Gleason score 7-8). Compared with the matched acinar adenocarcinoma control cases, the TMPRSS2-ERG gene fusion was significantly less frequently observed in ductal adenocarcinoma ( $P=0.002$, Fisher's exact test), as well as, in the acinar adenocarcinoma component of mixed ductalacinar adenocarcinoma tumors $(P=0.001$, Fisher's exact test).

\section{Discussion}

Perhaps the most compelling reason to subclassify prostatic carcinoma into ductal and acinar variants 
Table 3 TMPRSS2-ERG fusion status for ductal adenocarcinoma cases and their mixed acinar adenocarcinoma component and the spatial relationship between components

\begin{tabular}{|c|c|c|c|c|c|}
\hline Case \#: & Pathologic stage & Overall Gleason score & Ductal component & Acinar component & Spatial relationship \\
\hline 1 & TX NX MX & $\mathrm{U}$ & $\mathrm{D}$ & & \\
\hline 2 & TX NX MX & $\mathrm{U}$ & $\mathrm{N}$ & & \\
\hline 3 & TX NX MX & $\mathrm{U}$ & $\mathrm{N}$ & & \\
\hline 4 & T3A No MX & 7 & $\mathrm{~N}$ & & \\
\hline 5 & T3A No MX & 7 & $\mathrm{~N}$ & & \\
\hline 6 & T2 No MX & 7 & $\mathrm{~N}$ & $\mathrm{~N}$ & Commingled \\
\hline 7 & TX NX MX & $\mathrm{U}$ & $\mathrm{N}$ & $\mathrm{N}$ & Unknown \\
\hline 8 & T3B No MX & 7 & $\mathrm{~N}$ & $\mathrm{~N}$ & Commingled \\
\hline 9 & T2 No MX & 7 & $\mathrm{~N}$ & $\mathrm{~N}$ & Commingled \\
\hline 10 & T3A No MX & 7 & $\mathrm{D}$ & & \\
\hline 11 & T3B No MX & 8 & $\mathrm{~N}$ & & \\
\hline 12 & T3A No MX & 8 & $\mathrm{~N}$ & $\mathrm{~N}$ & Unknown \\
\hline 13 & T3A No MX & 8 & $\mathrm{~N}$ & & \\
\hline 14 & T3B No MX & 7 & $\mathrm{~N}$ & & \\
\hline 15 & T3A No MX & 7 & $\mathrm{~N}$ & $\mathrm{~N}$ & Separate \\
\hline 16 & T3A No MX & 7 & $\mathrm{~T}$ & $\mathrm{~T}$ & Commingled \\
\hline 17 & T2 No MX & 7 & $\mathrm{~N}$ & $\mathrm{~N}$ & Commingled \\
\hline 18 & TX No MX & 7 & $\mathrm{~N}$ & $\mathrm{~N}$ & Commingled \\
\hline 19 & T3A No MX & 7 & $\mathrm{~N}$ & & \\
\hline 20 & T2 No MX & 7 & $\mathrm{~N}$ & & \\
\hline 21 & T2 No MX & 8 & $\mathrm{~N}$ & $\mathrm{~N}$ & Commingled \\
\hline 22 & T2 No MX & 8 & $\mathrm{~N}$ & $\mathrm{~N}$ & Commingled \\
\hline 23 & T2 No MX & 7 & $\mathrm{~N}$ & & \\
\hline 24 & T2 No MX & 8 & $\mathrm{~N}$ & $\mathrm{~N}$ & Unknown \\
\hline 25 & T3A No MX & 7 & $\mathrm{~N}$ & & \\
\hline 26 & T3A No MX & 8 & $\mathrm{~N}$ & & \\
\hline 27 & T3B N1 MX & 9 & $\mathrm{~N}$ & $\mathrm{~N}$ & Commingled \\
\hline 28 & TX No MX & 7 & $\mathrm{~N}$ & & \\
\hline 29 & T3A No MX & 7 & $\mathrm{~N}$ & $\mathrm{~N}$ & Separate \\
\hline 30 & T3A No MX & 8 & $\mathrm{D}$ & $\mathrm{N}$ & Separate \\
\hline 31 & T2 No MX & 7 & $\mathrm{~N}$ & $\mathrm{~N}$ & Commingled \\
\hline 32 & T2 No MX & 7 & $\mathrm{~N}$ & $\mathrm{~N}$ & Separate \\
\hline 33 & T2 No MX & 7 & $\mathrm{~N}$ & & \\
\hline 34 & T2 No MX & 8 & $\mathrm{~N}$ & $\mathrm{~N}$ & Commingled \\
\hline 35 & T2 No MX & 8 & $\mathrm{~N}$ & $\mathrm{~N}$ & Commingled \\
\hline 36 & T3B N1 MX & 8 & $\mathrm{~N}$ & $\mathrm{~N}$ & Commingled \\
\hline 37 & T3B No MX & 9 & $\mathrm{~N}$ & $\mathrm{~N}$ & Commingled \\
\hline 38 & T3A No MX & 7 & $\mathrm{~N}$ & & \\
\hline
\end{tabular}

$\mathrm{D}=$ fusion by deletion, $\mathrm{T}=$ fusion by translocation, $\mathrm{N}=$ normal .

is that ductal adenocarcinoma has been found to have a clinically more aggressive course than the typical Gleason pattern 3 acinar adenocarcinoma. ${ }^{4-6}$ Although many of the original studies were conducted in pre-PSA era cohorts, a handful of more recent studies based on prostate needle biopsy with follow-up radical prostatectomy have shown similar results. ${ }^{7}$ Only rare reports have suggested that ductal adenocarcinoma may have a less aggressive course than typical acinar adenocarcinoma. ${ }^{14}$ However, because of the infrequent occurrence of ductal adenocarcinoma, studies with clinical follow-up of large cohorts, as seen in acinar adenocarcinoma, have not occurred. Although only acinar adenocarcinoma is included in Gleason's prostate carcinoma grading system, the current consensus is to treat ductal adenocarcinoma as if it is a Gleason $4+4=8$ carcinoma. ${ }^{15}$ This is in part based on evidence from a needle biopsy study that suggested that the prognosis of surgically resected ductal adenocarcinoma cases was between that of a Gleason score 7 and Gleason score 8 acinar adenocarcinoma. ${ }^{7}$
Despite these important clinical differences between ductal and acinar adenocarcinoma, to our knowledge, the two tumor types have not been compared on a molecular-genetic level. Although ductal adenocarcinoma and acinar adenocarcinoma may occur together in as many as $5 \%$ of radical prostatectomies, no prior studies have examined whether there is a clonal relationship between these tumor components. Based on morphologic observations, the ductal and acinar tumor components in mixed prostate carcinoma cases are more often commingled intimately than geographically separate; a finding that would suggest that these components are likely genetically related, at least in a subset of cases. ${ }^{16}$ In this study, we also found that in mixed ductal-acinar tumors, the two components were intermingled. Further, the very presence of the TMPRSS2-ERG rearrangement in $11 \%$ of the ductal adenocarcinoma cases suggests that at least some cases with the morphologic features of ductal adenocarcinoma may share some of the same early pathogenetic aberrations with acinar adeno- 
carcinoma cases. Additionally, in 95\% of the mixed ductal-acinar adenocarcinoma cases there was concordance for presence/absence of the TMPRSS2-ERG rearrangement between the ductal and acinar components of the tumor in a given case. As the vast majority of these cases did not have the TMPRSS2ERG rearrangement, this data certainly does not prove a genetic relationship between the acinar and ductal tumor components; however, it is not inconsistent with this hypothesis.

To date, the role of the TMPRSS2-ERG gene rearrangement in the initiation and progression of prostate carcinoma remains unclear. Early studies of the rearrangement suggested that the presence of the gene fusion was associated with higher stage and Gleason grade disease, and shorter interval to biochemical recurrence. ${ }^{17-20}$ However, recent larger studies in surgical cohorts have consistently found an inverse relationship between Gleason grade and rates of gene fusion, and no consistent relationship with prognosis, measured either by biochemical recurrence or overall survival has emerged. ${ }^{21,22} E R G$ over expression in transgenic mouse models causes prostatic intraepithelial neoplasia, but not invasive cancer, suggesting that TMPRSS2-ERG fusion may not be sufficient for transformation to an invasive phenotype. ${ }^{23}$ In vitro experiments using ERG over expression or knockdown in cell lines suggest that ERG may play a role in the activation of multiple cellular programs modulating tumor cell invasion, as well as in the activation of the oncogene $C-M Y C^{23-25}$ However, no dramatic role for the TMPRSS2-ERG fusion in prostate tumor initiation or progression has emerged.

Even in light of the inverse relationship with Gleason grade, the finding in this study that only $11 \%$ of the ductal adenocarcinoma cases showed the rearrangement is somewhat surprising. Most large studies of acinar adenocarcinoma have found the percent of rearranged cases to hover around $40-50 \%$, with higher Gleason score cases showing a minimum of $25 \%$ fusion rates in one study. ${ }^{17-19,21,22,26,27}$ In our PSA-era cohort of gradematched pure acinar adenocarcinoma controls (Gleason 7-8), we found $45 \%$ of cases had the rearrangement, a somewhat higher fraction than reported by Fine et al for the same grade, but one that is comparable with the overall rates of fusion reported in most surgical cohorts. Compared with our grade-matched pure acinar adenocarcinoma controls, the finding of only $11 \%$ rearrangement in the ductal carcinoma group is highly significant. Given that a subset of our pure acinar adenocarcinoma controls were embedded in the same ductal adenocarcinoma tissue microarray block, and these cases showed a much higher rate of TMPRSS2-ERG rearrangement, it appears unlikely that the low rate of rearrangement in the ductal adenocarcinoma cases is an artifact related to FISH or tissue microarray preparation.

Ultimately, the biologic explanation for the low rate of TMPRSS2-ERG fusions in prostatic ductal carcinoma requires further study, and may not emerge until we have a better understanding of the role of the fusion in prostate cancer initiation and progression. At the very least, our findings support the emerging concept that TMPRSS2-ERG fusion is less common in prostate tumors that appear more aggressive by traditional pathologic measures (ie, Gleason grade). Whether this genetic difference ever translates into an independent predictor of clinical outcome remains unclear. Similarly, how this inverse relationship between Gleason grade and frequency of TMPRSS2-ERG rearrangement can be explained, by what we know about the biological activity of $E R G$, is not evident. Here, of note, the frequency of TMPRSS2-ERG rearrangement in the acinar component of the mixed tumors in our series was also significantly lower than the frequency observed in the pathologic grade-matched pure acinar adenocarcinomas (45 vs 5\%). This may be additional, though indirect, evidence for a genetic relationship between the acinar and ductal components in mixed tumors. Further, this data suggests that the pathogenetic events leading to mixed ductal-acinar tumors may be distinct from those leading to pure acinar adenocarcinomas. Such differences may have important biologic and clinical relevance when the pathogenic role of the TMPRSS2-ERG gene rearrangement is more fully understood.

\section{Acknowledgement}

Funding for this research was provided by the Patrick C Walsh Prostate Cancer Research Fund.

\section{Conflicts of interest}

The authors have no disclosures/conflicts of interest to declare.

\section{References}

1 Epstein JI, Woodruff JM. Adenocarcinoma of the prostate with endometrioid features. A light microscopic and immunohistochemical study of ten cases. Cancer 1986;57:111-119.

2 Bock BJ, Bostwick DG. Does prostatic ductal adenocarcinoma exist? Am J Surg Pathol 1999;23:781-785.

3 Melicow MM, Pachter MR. Endometrial carcinoma of proxtatic utricle (uterus masculinus). Cancer 1967;20:1715-1722.

4 Greene LF, Farrow GM, Ravits JM, et al. Prostatic adenocarcinoma of ductal origin. J Urol 1979;121: 303-305.

5 Bostwick DG, Kindrachuk RW, Rouse RV. Prostatic adenocarcinoma with endometrioid features. Clinical, pathologic, and ultrastructural findings. Am J Surg Pathol 1985;9:595-609. 
6 Christensen WN, Steinberg G, Walsh PC, et al. Prostatic duct adenocarcinoma. Findings at radical prostatectomy. Cancer 1991;67:2118-2124.

7 Brinker DA, Potter SR, Epstein JI. Ductal adenocarcinoma of the prostate diagnosed on needle biopsy: correlation with clinical and radical prostatectomy findings and progression. Am J Surg Pathol 1999;23:1471-1479.

8 Orihuela E, Green JM. Ductal prostate cancer: contemporary management and outcomes. Urol Oncol 2008;26:368-371.

9 Tomlins SA, Rhodes DR, Perner S, et al. Recurrent fusion of TMPRSS2 and ETS transcription factor genes in prostate cancer. Science 2005;310:644-648.

10 Mehra R, Tomlins SA, Shen R, et al. Comprehensive assessment of TMPRSS2 and ETS family gene aberrations in clinically localized prostate cancer. Mod Pathol 2007;20:538-544.

11 Perner S, Demichelis F, Beroukhim R, et al. TMPRSS2:ERG fusion-associated deletions provide insight into the heterogeneity of prostate cancer. Cancer Res 2006;66:8337-8341.

12 Hessels D, Smit FP, Verhaegh GW, et al. Detection of TMPRSS2-ERG fusion transcripts and prostate cancer antigen 3 in urinary sediments may improve diagnosis of prostate cancer. Clin Cancer Res 2007;13:5103-5108.

13 Mosquera JM, Perner S, Genega EM, et al. Characterization of TMPRSS2-ERG fusion high-grade prostatic intraepithelial neoplasia and potential clinical implications. Clin Cancer Res 2008;14:3380-3385.

14 Millar EK, Sharma NK, Lessells AM. Ductal (endometrioid) adenocarcinoma of the prostate: a clinicopathological study of 16 cases. Histopathology 1996;29:11-19.

15 Epstein JI, Allsbrook WC, Amin MB, et al. Update on the Gleason grading system for prostate cancer: results of an international consensus conference of urologic pathologists. Adv Anat Pathol 2006;13:57-59.

16 Epstein JI, Netto GN. Biopsy Interpretation of the Prostate 2nd ed Lippincott, Williams \& Wilkins: Philadelphia, PA, 2008.

17 Perner S, Demichelis F, Beroukhim R, et al. TMPRSS2:ERG fusion-associated deletions provide insight into the heterogeneity of prostate cancer. Cancer Res 2006;66:8337-8341.

18 Mehra R, Tomlins SA, Shen R, et al. Comprehensive assessment of TMPRSS2 and ETS family gene aberrations in clinically localized prostate cancer. Mod Pathol 2007;20:538-544.

19 Nam RK, Sugar L, Yang W, et al. Expression of the TMPRSS2:ERG fusion gene predicts cancer recurrence after surgery for localised prostate cancer. Br J Cancer 2007;97:1690-1695.

20 Demichelis F, Fall K, Perner S, et al. TMPRSS2:ERG gene fusion associated with lethal prostate cancer in a watchful waiting cohort. Oncogene 2007;26: 4596-4599.

21 Gopalan A, Leversha M, Satagopan JM, et al. Increased copy number of TMPRSS2-ERG fusion, but not translocation alone, is associated with aggressive disease in patients treated by prostatectomy. Mod Pathol 2008;21:158A.

22 Fine SW, Gopalan A, Al-Ahmadie HA, et al. Does TMPRSS2-ERG gene fusion status in prostate cancer correlate with Gleason score? Mod Pathol 2008;21:156A.

23 Klezovitch O, Risk M, Coleman I, et al. A causal role for ERG in neoplastic transformation of prostate epithelium. Proc Natl Acad Sci USA 2008;105: 2105-2110.

24 Tomlins SA, Laxman B, Varambally S, et al. Role of the TMPRSS2-ERG gene fusion in prostate cancer. Neoplasia 2008;10:177-188.

25 Sun C, Dobi A, Mohamed A, et al. TMPRSS2-ERG fusion, a common genomic alteration in prostate cancer activates C-MYC and abrogates prostate epithelial differentiation. Oncogene 2008;27:5348-5353.

26 Wang J, Cai Y, Ren C, et al. Expression of variant TMPRSS2/ERG fusion messenger RNAs is associated with aggressive prostate cancer. Cancer Res 2006;66:8347-8351.

$27 \mathrm{Tu}$ JJ, Rohan S, Kao J, et al. Gene fusions between TMPRSS2 and ETS family genes in prostate cancer: frequency and transcript variant analysis by RT-PCR and FISH on paraffin-embedded tissues. Mod Pathol 2007;20:921-928. 